\title{
Utilization trends in inpatient endoscopic retrograde cholangiopancreatography (ERCP): A cross-sectional US experience
}

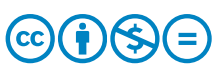

\section{Authors}

Moiz Ahmed ${ }^{1}$, Ritesh Kanotra ${ }^{2}$, Ghanshyambhai T. Savani ${ }^{3}$, Fenilkumar Kotadiya ${ }^{4}$, Nileshkumar Patel ${ }^{5}$, Sarah Tareen ${ }^{6}$, Matthew J. Fasullo ${ }^{7}$, Mayurathan Kesavan ${ }^{8}$, Ahsan Kahn ${ }^{8}$, Nikhil Nalluri ${ }^{1}$, Hafiz M. Khan ${ }^{1}$, Dhaval Pau' ${ }^{1}$, Jeffrey Abergel ${ }^{8}$, Liliane Deeb ${ }^{8}$, Sherif Andrawes ${ }^{8}$, Ananya Das ${ }^{9}$

\section{Institutions}

1 Department of Internal Medicine, Staten Island University Hospital, Northwell Health, Staten Island, New York, United States

2 Department of Internal Medicine, Banner Baywood Medical Center, Mesa, Arizona, USA

3 Prince George's Hospital Center, Cheverly, Maryland, United States

4 Anatomy Department, M. P. Shah Government Medical College, India

Department of Cardiology, University of Miami Miller School of Medicine/Jackson Memorial Hospital, Florida, United States

6 Institute of Clinical Research India (ICRI), Bangalore, India

7 Touro College of osteopathic Medicine, New York, United States

8 Department of Gastroenterology, Staten Island University hospital, Northwell Health, Staten Island, United States

9 St. Joseph Hospital Medical center, Phoenix, Arizona, USA/ Arizona Center for Digestive health, Gastroenterology, Gilbert, Arizona, United States

submitted 12.7 .2016

accepted after revision $\quad 30.12 .2016$
Bibliography

DOI http://dx.doi.org/10.1055/s-0043-102402 |

Endoscopy International Open 2017; 05: E261-E271

(c) Georg Thieme Verlag KG Stuttgart · New York

ISSN 2364-3722

Corresponding author

Ritesh Kanotra, 1139 W Spur Avenue, Gilbert, AZ 85233

Fax: +1-646-515-8435

riteshkanotra@gmail.com

\section{ABSTRACT}

Study aims The goal of our study was to determine the current trends for inpatient utilization for endoscopic retrograde cholangiopancreatography (ERCP) and its economic impact in the United States between 2002 and 2013.

Patients and methods A Nationwide Inpatient Sample from 2002 through 2013 was examined. We identified ERCPs using International Classification of Diseases (ICD-9) codes; Procedure codes $51.10,51.11,52.13,51.14,51.15,52.14$ and 52.92 for diagnostic and $51.84,51.86,52.97$ were studied. Rate of inpatient ERCP was calculated. The trends for therapeutic ERCPs were compared to the diagnostic ones. We analyzed patient and hospital characteristics, length of hospital stay, and cost of care after adjusting for weighted samples. We used the Cochran-Armitage test for categorical variables and linear regression for continuous variables.

Results A total of 411,409 ERCPs were performed from 2002 to 2013. The mean age was $59 \pm 19$ years; $61 \%$ were female and $57 \%$ were white. The total numbers of ERCPS increased by $12 \%$ from 2002 to 2011 , which was followed by a $10 \%$ decrease in the number of ERCPs between 2011 and 2013.

There was a significant increase in therapeutic ERCPs by $37 \%$, and a decrease in diagnostic ERCPs by 57\% from 2002 to 2013. Mean length of stay was 7 days $(S E=0.01)$ and the mean cost of hospitalization was $\$ 20,022$ ( $S E=41)$.

Conclusions Our large cross-sectional study shows a significant shift in ERCPs towards therapeutic indications and a decline in its conventional diagnostic utility. Overall there has been a reduction in inpatient ERCPs.

\section{Introduction}

ERCP is a primary modality for management of pancreatico-biliary disorders and also remains a superior means of diagnosing pancreatic malignancy, especially ampullary cancer. Over the last 2 decades invention of noninvasive imaging modalities such as MRCP, high-resolution computed tomography (CT), ultrasound (US) and endoscopic ultrasound (EUS) has increased
[1]. Current guidelines from the American Society of Gastrointestinal Endoscopy describe ERCP as primarily a therapeutic procedure [2]. There is a paucity of data in literature on use of inpatient ERCP after the early $2000 \mathrm{~s}$.

Our primary aim was to evaluate national trends in total diagnostic and therapeutic inpatient ERCPs in the United States between the years 2002 and 2013. 
In view of today's health system in the United States, which is a bundled single-payor system based on a patient's acute and post-acute care, reduction in the length of hospitalization and cost of care is of utmost importance to hospitals all over the country [3]. There are very little data to date examining the inpatient cost and length of stay (LOS) for patients who have undergone inpatient ERCP. The Secondary goal of our study was to determine the economic impact of ERCPs on the health care system in the United States, by estimating the LOS and cost for ERCP-related hospitalizations over the same time frame.

\section{Patients and methods}

\section{Data source}

The National Inpatient Sample (NIS) was created by the Agency for Healthcare Research and Quality, which is the largest allpayer inpatient database available in the United States. This constitutes all discharge data across 45 states [4]. These data hav been used in the past to analyze national trends in outcomes for various diagnoses and major procedures; hospitalization rates, healthcare access and disparity of care. The NIS represents approximately $20 \%$ of US community hospitals and they provide sampling weights to project national estimates. Each individual hospitalization is deidentified and maintained as a unique entry with a primary discharge diagnosis and fewer than 24 secondary diagnoses. It also contains information on demographics, comorbidities, insurance status, primary and secondary procedures, hospitalization outcome, LOS and cost of care, with safeguards to protect patient, physician and hospital privacy. Note that the variables of hospital location, region and teaching status were not reported in the dataset for the year 2012-2013. The NIS data have been compared against data sources from The American Hospital Association Annual Survey Database, The National Hospital Discharge Survey from The National Center for Health Statistics, and MedPAR inpatient data from the Centers for Medicare and Medicaid Services. Furthermore, annual data quality assessments are performed, thus guaranteeing the external and internal validity of the database.

\section{Study population}

Using the NIS we identified in-hospital ERCPs from 2002 through 2013 using the International Classification of Disease (ICD), $9^{\text {th }}$ revision, clinical modification procedural codes 51.10 (ERCP), 51.11 (ERC), 52.13 (ERP), 51.14 (endoscopic biopsy of biliary duct or sphincter of oddi), 51.15 (Pressure measurement of sphincter of oddi), 52.14 (endoscopic biopsy of pancreatic duct) and 52.92 (Cannulation of pancreatic duct) for diagnostic and 51.84 (Endoscopic dilation of ampulla and biliary duct), 51.86 (Endoscopic insertion of nasobiliary drainage tube), 52.97 (Endoscopic insertion of nasopancreatic drainage tube), 51.88 (Endoscopic removal of stone from biliary tract), 52.93 (Endoscopic insertion of stent into pancreatic duct), 51.85 (Endoscopic sphincterotomy and papillotomy), 51.64 (Endoscopic excision or destruction of lesion of biliary ducts or sphincter of Oddi), 52.21 (Endoscopic excision or destruction of lesion or tissue of pancreatic duct), 51.69 (Excision of other bile duct), 51.87 (Endoscopic insertion of stent into bile duct),
52.94 (Endoscopic removal of stone from pancreatic duct) and 52.98 (Endoscopic dilation of pancreatic duct) for therapeutic ERCPs. Gall bladder and biliary disease were identified using ICD-9 codes 577 and all patients older than 18 years were included. Those with missing age, sex, admission or discharge date were excluded.

\section{Definition of variables}

NIS variables were used to identify patient demographics. Hospitals with an Accreditation Council for Graduate Medical Education (ACGME)-accredited residency program, which were members of the Council of Teaching Hospitals, and/or had fulltime equivalent interns and a resident-to-patient ratio $\geq 0.25$ were considered as teaching hospitals. Hospital location (rural/ urban) and bed size were also taken into account. Because the NIS represents $20 \%$ of the stratified US hospital sample, utilization rates were calculated by dividing the number of ERCP procedures performed, available in the NIS dataset, in a given year divided by $20 \%$ of the US census population aged 18 years or older for that year. Deyo modification of the Charlson Comorbidity Index $(\mathrm{CCl})$ was used to define the severity of comorbid conditions. That contains 17 comorbid conditions with differential weights, with scores ranging from 0 to 33 . The LOS for each hospitalization was calculated after excluding those who died during their stay. Cost of hospitalization $(\mathrm{COH})$ was determined after merging data with cost-to-charge ratio files available from the Healthcare Cost and Utilization Project (HCUP) website. The total cost of each hospitalization was determined by multiplying the cost-to-charge ratio with the total hospital charge. Inflation was accounted for by adjusting the cost of each year in reference to the 2016 US dollar value using Consumer Price Index data.

\section{Statistical analysis}

SAS 9.4 (SAS Institute Inc, Cary, North Carolina) was used for the analyses. Nationally representative estimates were produced using the weight variable provided by the NIS. The categorical variables are expressed in terms of the percentage (\%) of the total study population while continuous variables are expressed in terms of mean with its standard error. For trend analysis, we used the Cochran-Armitage test for categorical variables and linear regression for continuous variables. A $P$ value < 0.05 was considered statistically significant.

To identify significant predictors of length of stay and cost, we generated 2-level hierarchical mixed effects linear regression models (with patient-level factors nested within hospitallevel factors) with the unique hospital identification number incorporated as random effects within the model. In each multivariate model, we included the following patient-level and hospital-level variables: age (per 10-year change), gender, Deyo modification of Charlson comorbidity index, primary payer, median household income category (as per patient's residential zip code), admission type (elective vs. non-elective), weekend admissions, hospital bed-size category (as per hospital's location and number of beds), hospital region (Midwest, South or West with Northeast as referent), hospital location (rural vs. urban) and hospitas teaching status (teaching vs. non-teaching). 


\section{Results}

A total of 411,409 inpatient ERCP procedures were performed throughout the United States between 2002 and 2013. Overall inpatient ERCPs increased from 34,054 in 2002 to 38,112 in 2011. This was followed by a drastic drop to 34,131 in 2013. As shown in $>$ Table 1 , the mean age of the patients was $59 \pm 19$ years, majority females (61\%) and whites (57\%). There was a $17 \%$ relative increase in ERCPs among the Hispanic population between 2002 and 2013. Most of the patients had a Charlson $(\mathrm{CCl})$ score of 0 (50\%). The primary payer was largely Medicare (45\%) and private insurance (33\%). Most of the admissions were seen in large hospitals (66\%) at urban (77\%) centers. Almost all of the admissions for the procedure occurred during the weekday (79\%).

Of all the ERCPs admissions, 283,552 (69\%) had therapeutic ERCPs, 82,522 (20\%) were coded for diagnostic-only ERCPs and the remaining 45,335 (11\%) were coded as combined diagnostic and therapeutic ERCPs. There was an increase in the number of therapeutic ERCPs from 19,256 in 2002 to 29,411 in 2011 followed by a fall to 26,187 cases in 2012, which then plateaued at 26,418 in 2013 ( $P$ for trend $<0.001)$. A significant decrease in the diagnostic utility of ERCPs was noticed from 10,508 in 2002 to 4,496 in 2013 ( $P$ for trend $<0.001$ ) ( $\triangleright$ Fig. 1 ). The most common procedure coded was endoscopic sphincterotomy (43\%) followed by endoscopic biliary stone removal (29\%) and endoscopic biliary stenting (21\%). Some of the most commonly coded diagnosis over the years included pancreatitis (14\%), biliary obstruction (12\%), and cholangitis (12\%) ( Table 2). The most common ERCP-related complications coded for included hemorrhage hematoma (3\%), respiratory failure $(1.4 \%)$ and hemorrhage requiring transfusion (1.2\%) as seen in $>$ Table 3 .

The mean LOS for patients discharged post-ERCP was 7 days $(\mathrm{SE}=0.01)$. Charlson score $>2$, weekend admission ( $\triangleright$ Fig. 2 ) admission to a large hospital, teaching hospital, and urban location were strongly predictive of extended LOS. The mean LOS was significantly higher in 2002 at 7.4 days $(S E=0.01)$ as compared to 6.6 days $(\mathrm{SE}=0.02)$ in 2013 ( $P$ for trend $<0.001)$ ( $\triangleright \mathrm{Ta}$ ble 4).

After adjusting to 2016 dollars, the mean cost of hospitalization during the study period was $\$ 20,022$ ( $S E=41)$. Note that patients with Charlson score > 2, weekend admission ( $>$ Fig.3), nad urban and teaching hospitals were independent predictors of increased cost of hospitalization during the procedure. On the contrary, elective admission and private insurance payer were associated with decreased cost of care. We also found the cost to be lowest in the southern region of the United States followed by the Midwest, West and Northeast, respectively. The mean cost significantly decreased from \$19,190 (SE

- Table 1 Baseline characteristics.

\begin{tabular}{|c|c|c|c|c|c|c|c|c|c|}
\hline Year & Overall & $\begin{array}{l}2002- \\
2003\end{array}$ & $\begin{array}{l}2004- \\
2005\end{array}$ & $\begin{array}{l}2006- \\
2007\end{array}$ & $\begin{array}{l}2008- \\
2009\end{array}$ & $\begin{array}{l}2010- \\
2011\end{array}$ & $\begin{array}{l}2012- \\
2013\end{array}$ & $\begin{array}{l}\text { Chi } \\
\text { square } \\
P \text { value }\end{array}$ & $\begin{array}{l}P \text { value } \\
\text { for trend }\end{array}$ \\
\hline \multicolumn{10}{|c|}{ No. Of cases $=411,409$} \\
\hline \multicolumn{8}{|l|}{ Age } & $<0.001$ & \\
\hline $18-29$ & 9.8 & $9.50 \%$ & $9.47 \%$ & $10.02 \%$ & $10.23 \%$ & $10.13 \%$ & $9.52 \%$ & & 0.0160 \\
\hline $30-39$ & 9.6 & $10.00 \%$ & $9.45 \%$ & $9.87 \%$ & $9.59 \%$ & $9.57 \%$ & $9.34 \%$ & & 0.0006 \\
\hline $40-49$ & 11.9 & $12.53 \%$ & $12.52 \%$ & $11.97 \%$ & $11.76 \%$ & $11.51 \%$ & $10.89 \%$ & & $<0.001$ \\
\hline $50-59$ & 15.5 & $14.74 \%$ & $15.20 \%$ & $15.45 \%$ & $15.40 \%$ & $15.90 \%$ & $16.08 \%$ & & $<0.001$ \\
\hline $60-69$ & 16.7 & $15.33 \%$ & $15.57 \%$ & $15.81 \%$ & $17.06 \%$ & $17.65 \%$ & $18.45 \%$ & & $<0.001$ \\
\hline $70-79$ & 18.2 & $20.27 \%$ & $19.11 \%$ & $18.18 \%$ & $17.62 \%$ & $16.91 \%$ & $17.39 \%$ & & $<0.001$ \\
\hline$>=80$ & 18.3 & $17.64 \%$ & $18.68 \%$ & $18.70 \%$ & $18.34 \%$ & $18.34 \%$ & $18.32 \%$ & & 0.0892 \\
\hline \multicolumn{8}{|l|}{ Sex } & $<0.001$ & \\
\hline Male & 39.0 & $37.59 \%$ & $38.23 \%$ & $38.31 \%$ & $38.89 \%$ & $39.84 \%$ & $40.96 \%$ & & \multirow[t]{2}{*}{$<0.001$} \\
\hline Female & 61.0 & $62.41 \%$ & $61.77 \%$ & $61.69 \%$ & $61.11 \%$ & $60.16 \%$ & $59.04 \%$ & & \\
\hline \multicolumn{8}{|l|}{ Race } & $<0.001$ & \\
\hline White & 57.1 & $51.65 \%$ & $53.74 \%$ & $51.60 \%$ & $58.66 \%$ & $62.37 \%$ & $63.90 \%$ & & $<0.001$ \\
\hline Black & 7.4 & $7.20 \%$ & $6.52 \%$ & $6.37 \%$ & $6.93 \%$ & $8.63 \%$ & $8.67 \%$ & & $<0.001$ \\
\hline Hispanic & 12.8 & $11.03 \%$ & $11.42 \%$ & $12.95 \%$ & $12.40 \%$ & $14.40 \%$ & $14.47 \%$ & & $<0.001$ \\
\hline Others & 5.9 & $4.87 \%$ & $4.83 \%$ & $5.44 \%$ & $6.80 \%$ & $6.23 \%$ & $7.41 \%$ & & $<0.001$ \\
\hline Missing & 16.7 & $25.26 \%$ & $23.50 \%$ & $23.64 \%$ & $15.21 \%$ & $8.37 \%$ & $5.56 \%$ & & $<0.001$ \\
\hline
\end{tabular}


- Table 1 (Continuation)

\begin{tabular}{|c|c|c|c|c|c|c|c|c|c|}
\hline Year & Overall & $\begin{array}{l}2002- \\
2003\end{array}$ & $\begin{array}{l}2004- \\
2005\end{array}$ & $\begin{array}{l}2006- \\
2007\end{array}$ & $\begin{array}{l}2008- \\
2009\end{array}$ & $\begin{array}{l}2010- \\
2011\end{array}$ & $\begin{array}{l}2012- \\
2013\end{array}$ & $\begin{array}{l}\text { Chi } \\
\text { square } \\
P \text { value }\end{array}$ & $\begin{array}{l}P \text { value } \\
\text { for trend }\end{array}$ \\
\hline \multicolumn{8}{|c|}{ Comorbidities (Charlson score) } & $<0.001$ & \\
\hline 0 & 50.7 & $55.46 \%$ & $53.55 \%$ & $51.31 \%$ & $49.68 \%$ & $47.64 \%$ & $46.97 \%$ & & $<0.001$ \\
\hline 1 & 20.3 & $20.60 \%$ & $21.39 \%$ & $20.51 \%$ & $19.76 \%$ & $19.74 \%$ & $19.70 \%$ & & $<0.001$ \\
\hline$\geq 2$ & 29.0 & $23.94 \%$ & $25.06 \%$ & $28.18 \%$ & $30.56 \%$ & $32.62 \%$ & $33.33 \%$ & & $<0.001$ \\
\hline \multicolumn{8}{|c|}{ Median household income } & $<0.001$ & \\
\hline 1st quartile & 23.3 & $14.89 \%$ & $25.23 \%$ & $25.67 \%$ & $24.21 \%$ & $25.91 \%$ & $27.02 \%$ & & $<0.001$ \\
\hline 2nd quartile & 24.4 & $22.71 \%$ & $25.27 \%$ & $24.84 \%$ & $26.22 \%$ & $24.90 \%$ & $25.45 \%$ & & $<0.001$ \\
\hline 3 rd quartile & 24.9 & $25.84 \%$ & $24.78 \%$ & $24.91 \%$ & $25.72 \%$ & $26.26 \%$ & $25.23 \%$ & & 0.1137 \\
\hline 4th quartile & 25.2 & $36.56 \%$ & $24.72 \%$ & $24.58 \%$ & $23.85 \%$ & $22.93 \%$ & $22.31 \%$ & & $<0.001$ \\
\hline \multicolumn{8}{|l|}{ Primary payer } & $<0.001$ & \\
\hline Medicare & 45.3 & $45.70 \%$ & $45.81 \%$ & $45.20 \%$ & $44.22 \%$ & $44.90 \%$ & $46.68 \%$ & & 0.4882 \\
\hline Medicaid & 11.2 & $10.36 \%$ & $10.51 \%$ & $10.62 \%$ & $10.98 \%$ & $12.42 \%$ & $12.22 \%$ & & $<0.001$ \\
\hline Private & 33.5 & $36.15 \%$ & $34.71 \%$ & $34.37 \%$ & $34.63 \%$ & $31.98 \%$ & $29.99 \%$ & & $<0.001$ \\
\hline Uninsured & 9.8 & $7.79 \%$ & $8.97 \%$ & $9.81 \%$ & $10.17 \%$ & $10.71 \%$ & $11.11 \%$ & & $<0.001$ \\
\hline \multicolumn{10}{|c|}{ Hospital characteristics } \\
\hline \multicolumn{8}{|c|}{ Hospital bed size } & $<0.001$ & \\
\hline Small & 9.4 & $8.90 \%$ & $8.61 \%$ & $10.88 \%$ & $9.40 \%$ & $8.93 \%$ & $9.91 \%$ & & $<0.001$ \\
\hline Medium & 24.2 & $25.50 \%$ & $25.98 \%$ & $24.45 \%$ & $22.83 \%$ & $21.96 \%$ & $25.02 \%$ & & $<0.001$ \\
\hline Large & 66.1 & $65.60 \%$ & $65.41 \%$ & $64.67 \%$ & $67.77 \%$ & $69.11 \%$ & $65.07 \%$ & & $<0.001$ \\
\hline \multicolumn{8}{|c|}{ Hospital location } & $<0.001$ & \\
\hline Rural & 5.5 & $9.00 \%$ & $6.46 \%$ & $6.14 \%$ & $5.88 \%$ & $5.54 \%$ & not reported & & \multirow[t]{2}{*}{$<0.001$} \\
\hline Urban & 77.6 & $91.00 \%$ & $93.54 \%$ & $93.86 \%$ & $94.12 \%$ & $94.46 \%$ & not reported & & \\
\hline \multicolumn{8}{|c|}{ Hospital region } & $<0.001$ & \\
\hline Northeast & 16.6 & $20.04 \%$ & $22.09 \%$ & $19.72 \%$ & $18.92 \%$ & $19.13 \%$ & Not reported & & $<0.001$ \\
\hline Midwest & 18.3 & $21.48 \%$ & $21.10 \%$ & $21.34 \%$ & $22.97 \%$ & $22.45 \%$ & Not reported & & $<0.001$ \\
\hline South & 29.8 & $37.31 \%$ & $36.06 \%$ & $34.95 \%$ & $34.84 \%$ & $35.47 \%$ & Not reported & & $<0.001$ \\
\hline West & 18.7 & $21.17 \%$ & $20.76 \%$ & $24.00 \%$ & $23.27 \%$ & $22.95 \%$ & Not reported & & $<0.001$ \\
\hline \multicolumn{8}{|c|}{ Hospital teaching status } & $<0.001$ & \\
\hline Nonteaching & 41.8 & $53.04 \%$ & $54.29 \%$ & $48.95 \%$ & $47.72 \%$ & $48.07 \%$ & Not reported & & \multirow[t]{2}{*}{$<0.001$} \\
\hline Teaching & 41.2 & $46.96 \%$ & $45.71 \%$ & $51.05 \%$ & $52.28 \%$ & $51.93 \%$ & Not reported & & \\
\hline \multicolumn{8}{|l|}{ Admission Day } & $<0.001$ & \\
\hline Weekday & $78.56 \%$ & $78.76 \%$ & $79.17 \%$ & $79.07 \%$ & $78.40 \%$ & $78.21 \%$ & $77.85 \%$ & & \multirow[t]{2}{*}{$<0.001$} \\
\hline Weekend & $21.44 \%$ & $21.24 \%$ & $20.83 \%$ & $20.93 \%$ & $21.60 \%$ & $21.79 \%$ & $22.15 \%$ & & \\
\hline $\begin{array}{l}\text { Cost (in } 2016 \\
\text { terms), } \\
\$-\text { mean } \\
\text { (std. error) }\end{array}$ & $20022(41)$ & $\begin{array}{l}19190 \\
(105)\end{array}$ & $\begin{array}{l}20350 \\
(113)\end{array}$ & $\begin{array}{l}20389 \\
(99)\end{array}$ & $\begin{array}{l}21013 \\
(107)\end{array}$ & $\begin{array}{l}20919 \\
(101)\end{array}$ & $18219(87)$ & & $<0.001$ \\
\hline $\begin{array}{l}\text { LOS, days - } \\
\text { mean } \\
\text { (std. error) }\end{array}$ & $6.97(0.01)$ & $7.38(0.03)$ & $7.42(0.03)$ & $7.14(0.03)$ & $6.93(0.03)$ & $6.62(0.02)$ & $6.38(0.02)$ & & $<0.001$ \\
\hline
\end{tabular}




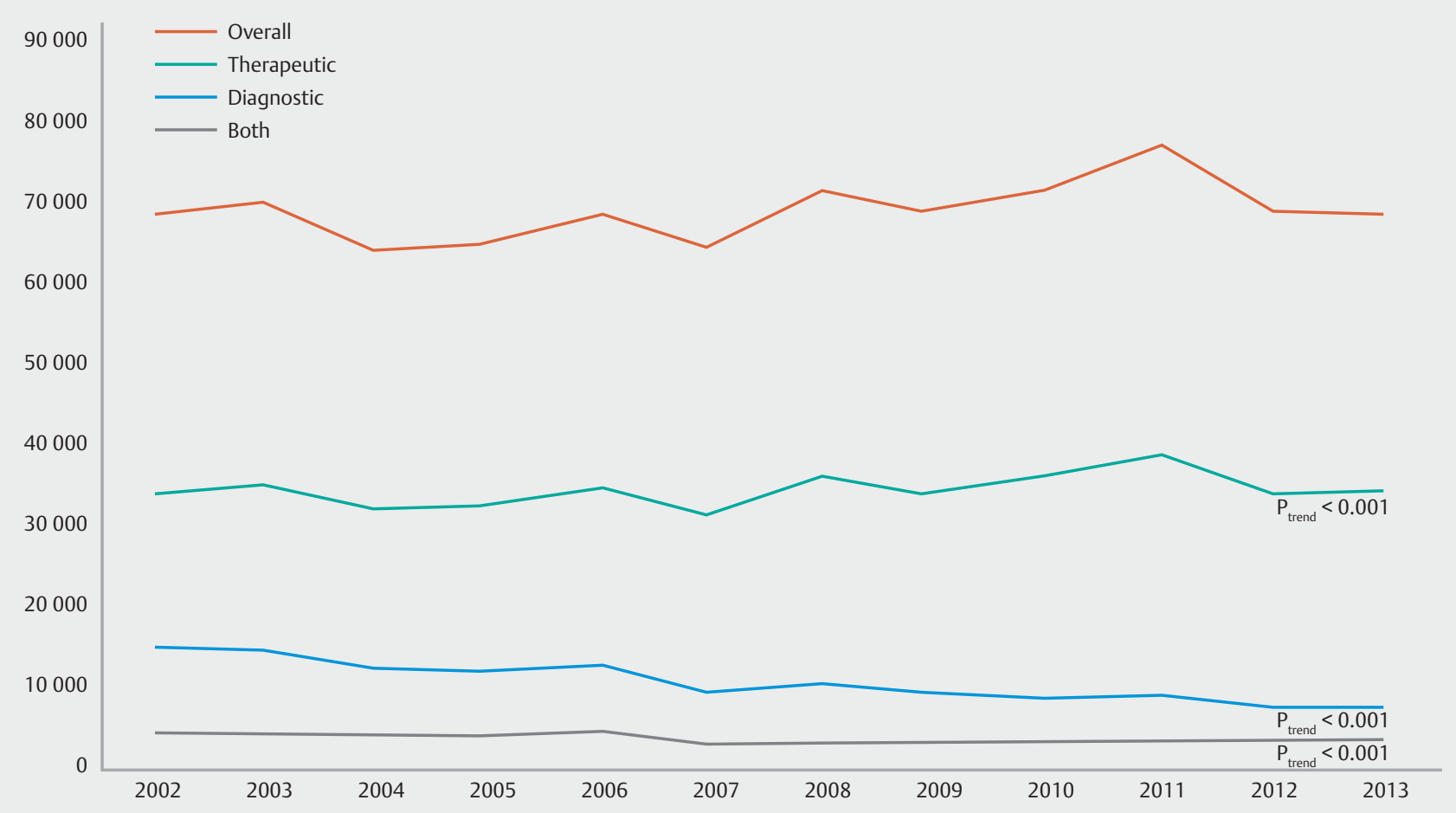

- Fig. 1 Trends for in-hospital diagnostic and therapeutic ERCPs throughout the United States ERCP, endoscopic retrograde cholangiopancreatography

- Table 2 Indications for ERCP.

\begin{tabular}{|c|c|c|c|c|c|c|c|c|}
\hline Indications & Overall & $\begin{array}{l}2002- \\
2003\end{array}$ & $\begin{array}{l}2004- \\
2005\end{array}$ & $\begin{array}{l}2006- \\
2007\end{array}$ & $\begin{array}{l}2008- \\
2009\end{array}$ & $\begin{array}{l}2010- \\
2011\end{array}$ & $\begin{array}{l}2012- \\
2013\end{array}$ & $\begin{array}{l}P \text { value } \\
\text { for Trend }\end{array}$ \\
\hline Endoscopic dilation & $4.41 \%$ & $3.42 \%$ & $3.66 \%$ & $3.69 \%$ & $4.27 \%$ & $5.28 \%$ & $6.05 \%$ & $<0.001$ \\
\hline Endoscopic sphincterotomy & $42.99 \%$ & $36.78 \%$ & $39.15 \%$ & $41.57 \%$ & $46.17 \%$ & $46.67 \%$ & $46.99 \%$ & $<0.001$ \\
\hline Endoscopic biliary stenting & $21.00 \%$ & $16.16 \%$ & $18.74 \%$ & $20.22 \%$ & $21.39 \%$ & $23.95 \%$ & $25.12 \%$ & $<0.001$ \\
\hline Endoscopic biliary stone removal & $29.33 \%$ & $25.02 \%$ & $26.95 \%$ & $28.63 \%$ & $30.11 \%$ & $32.75 \%$ & $32.08 \%$ & $<0.001$ \\
\hline Endoscopic pancreatic stent & $4.47 \%$ & $3.27 \%$ & $3.78 \%$ & $4.47 \%$ & $5.03 \%$ & $5.09 \%$ & $5.07 \%$ & $<0.001$ \\
\hline $\begin{array}{l}\text { Endoscopic pancreatic stone } \\
\text { removal }\end{array}$ & $0.24 \%$ & $0.18 \%$ & $0.18 \%$ & $0.22 \%$ & $0.26 \%$ & $0.29 \%$ & $0.31 \%$ & $<0.001$ \\
\hline $\begin{array}{l}\text { Endoscopic nasopancreatic } \\
\text { drainage }\end{array}$ & $0.02 \%$ & $0.01 \%$ & $0.02 \%$ & $0.02 \%$ & $0.03 \%$ & $0.01 \%$ & $0.02 \%$ & 0.638 \\
\hline $\begin{array}{l}\text { Endoscopic dilation pancreatic } \\
\text { duct }\end{array}$ & $0.37 \%$ & $0.27 \%$ & $0.31 \%$ & $0.37 \%$ & $0.35 \%$ & $0.44 \%$ & $0.45 \%$ & $<0.001$ \\
\hline Endoscopic manometry & $0.01 \%$ & $0.00 \%$ & $0.01 \%$ & $0.01 \%$ & $0.01 \%$ & $0.01 \%$ & $0.01 \%$ & $<0.001$ \\
\hline $\begin{array}{l}\text { Pancreatitis (indication \& } \\
\text { complication) }\end{array}$ & $14.07 \%$ & $11.88 \%$ & $12.86 \%$ & $13.48 \%$ & $14.99 \%$ & $15.49 \%$ & $15.52 \%$ & $<0.001$ \\
\hline $\begin{array}{l}\text { Cholangitis (indication \& } \\
\text { complication) }\end{array}$ & $11.61 \%$ & $9.20 \%$ & $9.90 \%$ & $10.46 \%$ & $11.86 \%$ & $13.00 \%$ & $14.98 \%$ & $<0.001$ \\
\hline Biliary obstruction & $12.06 \%$ & $9.15 \%$ & $9.83 \%$ & $10.33 \%$ & $14.25 \%$ & $14.04 \%$ & $14.34 \%$ & $<0.001$ \\
\hline Cholecystitis & $0.42 \%$ & $0.31 \%$ & $0.35 \%$ & $0.40 \%$ & $0.41 \%$ & $0.48 \%$ & $0.54 \%$ & $<0.001$ \\
\hline Spasm Of Sphincter Of Oddi (I) & $0.09 \%$ & $0.08 \%$ & $0.08 \%$ & $0.07 \%$ & $0.09 \%$ & $0.10 \%$ & $0.08 \%$ & 0.454 \\
\hline
\end{tabular}


- Table 2 (Continuation)

\begin{tabular}{|c|c|c|c|c|c|c|c|c|}
\hline Indications & Overall & $\begin{array}{l}2002- \\
2003\end{array}$ & $\begin{array}{l}2004- \\
2005\end{array}$ & $\begin{array}{l}2006- \\
2007\end{array}$ & $\begin{array}{l}2008- \\
2009\end{array}$ & $\begin{array}{l}2010- \\
2011\end{array}$ & $\begin{array}{l}2012- \\
2013\end{array}$ & $\begin{array}{l}P \text { value } \\
\text { for Trend }\end{array}$ \\
\hline Chronic pancreatitis & $3.13 \%$ & $2.42 \%$ & $2.55 \%$ & $2.91 \%$ & $3.28 \%$ & $3.78 \%$ & $3.77 \%$ & $<0.001$ \\
\hline Pancreatic cyst & $1.47 \%$ & $1.09 \%$ & $1.20 \%$ & $1.29 \%$ & $1.59 \%$ & $1.80 \%$ & $1.80 \%$ & $<0.001$ \\
\hline Deodenal neoplasm & $0.18 \%$ & $0.16 \%$ & $0.16 \%$ & $0.17 \%$ & $0.21 \%$ & $0.21 \%$ & $0.19 \%$ & 0.0136 \\
\hline Gallbladder neoplasm & $0.23 \%$ & $0.17 \%$ & $0.19 \%$ & $0.19 \%$ & $0.22 \%$ & $0.25 \%$ & $0.35 \%$ & $<0.001$ \\
\hline Extrahepatic neoplasm & $0.35 \%$ & $0.31 \%$ & $0.30 \%$ & $0.33 \%$ & $0.40 \%$ & $0.36 \%$ & $0.38 \%$ & 0.001 \\
\hline Ampula Of Vater cancer & $0.13 \%$ & $0.10 \%$ & $0.11 \%$ & $0.14 \%$ & $0.14 \%$ & $0.14 \%$ & $0.14 \%$ & 0.022 \\
\hline Other neoplasm Of gallbladder & $0.03 \%$ & $0.04 \%$ & $0.04 \%$ & $0.03 \%$ & $0.03 \%$ & $0.02 \%$ & $0.01 \%$ & $<0.001$ \\
\hline Pancreatic $t$ umor & $2.17 \%$ & $1.70 \%$ & $1.77 \%$ & $2.09 \%$ & $2.41 \%$ & $2.49 \%$ & $2.51 \%$ & $<0.001$ \\
\hline
\end{tabular}

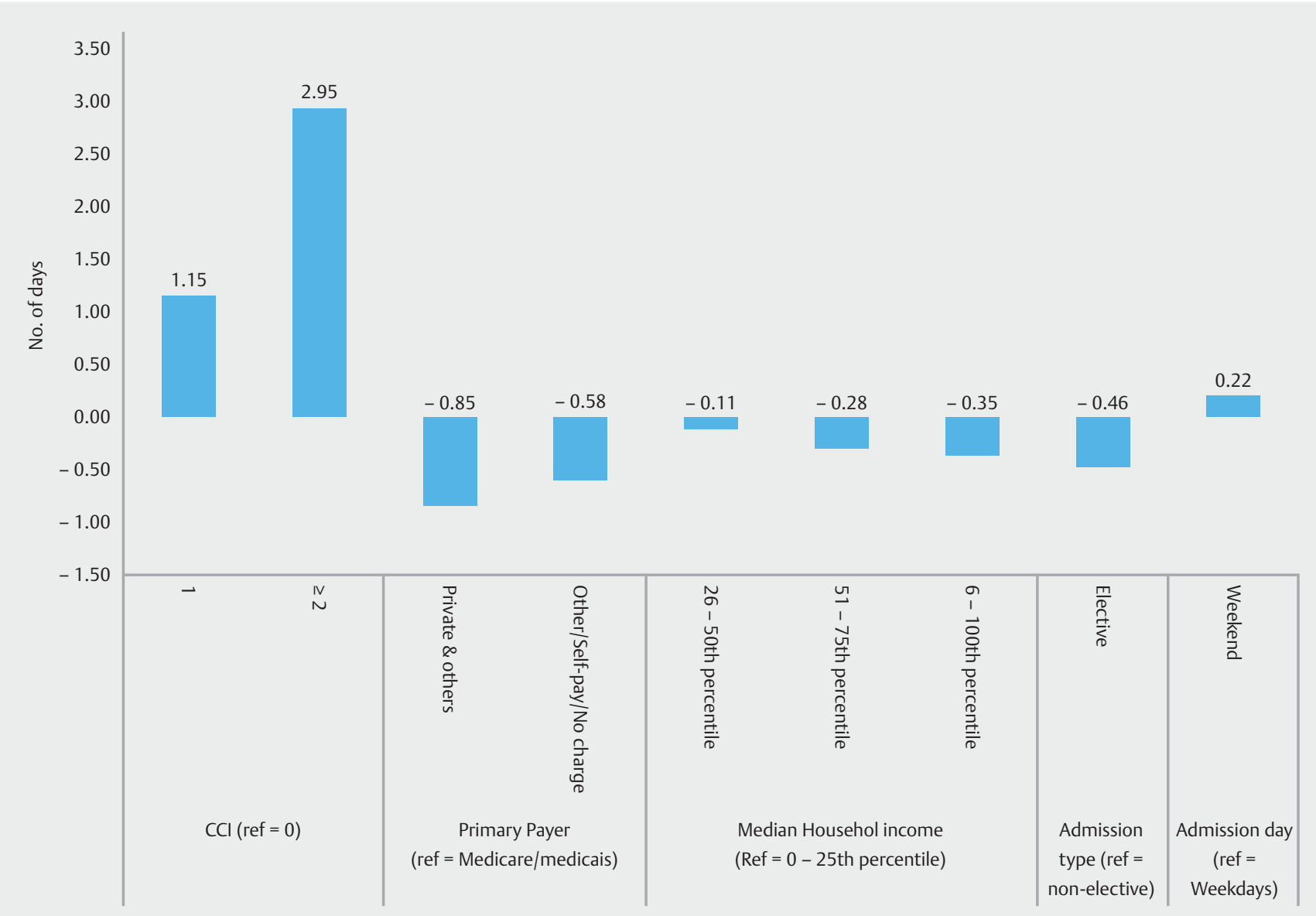

- Fig. 2 Multivariate predictors of length of hospital stay post-ERCP ERCP, endoscopic retrograde cholangiopancreatography 
> Table 3 ERCP-related complications.

\begin{tabular}{|c|c|c|c|c|c|c|c|c|}
\hline Complications & Overall & $\begin{array}{l}2002- \\
2003\end{array}$ & $\begin{array}{l}2004- \\
2005\end{array}$ & $\begin{array}{l}2006- \\
2007\end{array}$ & $\begin{array}{l}2008- \\
2009\end{array}$ & $\begin{array}{l}2010- \\
2011\end{array}$ & $\begin{array}{l}2012- \\
2013\end{array}$ & $\begin{array}{l}P \text { value } \\
\text { for Trend }\end{array}$ \\
\hline Biliary fistula & $0.23 \%$ & $0.25 \%$ & $0.23 \%$ & $0.24 \%$ & $0.22 \%$ & $0.20 \%$ & $0.24 \%$ & 0.424 \\
\hline Gall bladder perforation & $0.23 \%$ & $0.21 \%$ & $0.19 \%$ & $0.21 \%$ & $0.23 \%$ & $0.26 \%$ & $0.27 \%$ & $<0.001$ \\
\hline Gall bladder fistula & $0.07 \%$ & $0.08 \%$ & $0.08 \%$ & $0.06 \%$ & $0.06 \%$ & $0.07 \%$ & $0.06 \%$ & 0.081 \\
\hline Duodenal perforation & & $0.00 \%$ & $0.00 \%$ & $0.00 \%$ & $0.00 \%$ & $0.00 \%$ & $0.00 \%$ & \\
\hline Gastric perforation & $0.01 \%$ & $0.01 \%$ & $0.00 \%$ & $0.00 \%$ & $0.00 \%$ & $0.01 \%$ & $0.01 \%$ & 0.054 \\
\hline Upper GI bleed & & 0 & 0 & 0 & 0 & 0 & 0 & \\
\hline Hemorrhage hematoma & $3.13 \%$ & $2.39 \%$ & $2.48 \%$ & $2.58 \%$ & $3.16 \%$ & $3.87 \%$ & $4.16 \%$ & $<0.001$ \\
\hline $\begin{array}{l}\text { Hemorrhage requiring } \\
\text { transfusion }\end{array}$ & $1.21 \%$ & $0.66 \%$ & $0.87 \%$ & $0.98 \%$ & $1.33 \%$ & $1.64 \%$ & $1.73 \%$ & $<0.001$ \\
\hline Cardiovascular complications & $0.53 \%$ & $0.60 \%$ & $0.61 \%$ & $0.58 \%$ & $0.57 \%$ & $0.45 \%$ & $0.37 \%$ & $<0.001$ \\
\hline Respiratory failure & $1.40 \%$ & $1.04 \%$ & $1.16 \%$ & $1.32 \%$ & $1.33 \%$ & $1.36 \%$ & $2.16 \%$ & $<0.001$ \\
\hline Pneumothorax & $0.12 \%$ & $0.12 \%$ & $0.10 \%$ & $0.13 \%$ & $0.15 \%$ & $0.13 \%$ & $0.11 \%$ & 0.691 \\
\hline Pneumomediastinum & $0.02 \%$ & $0.02 \%$ & $0.02 \%$ & $0.02 \%$ & $0.01 \%$ & $0.02 \%$ & $0.01 \%$ & 0.518 \\
\hline
\end{tabular}

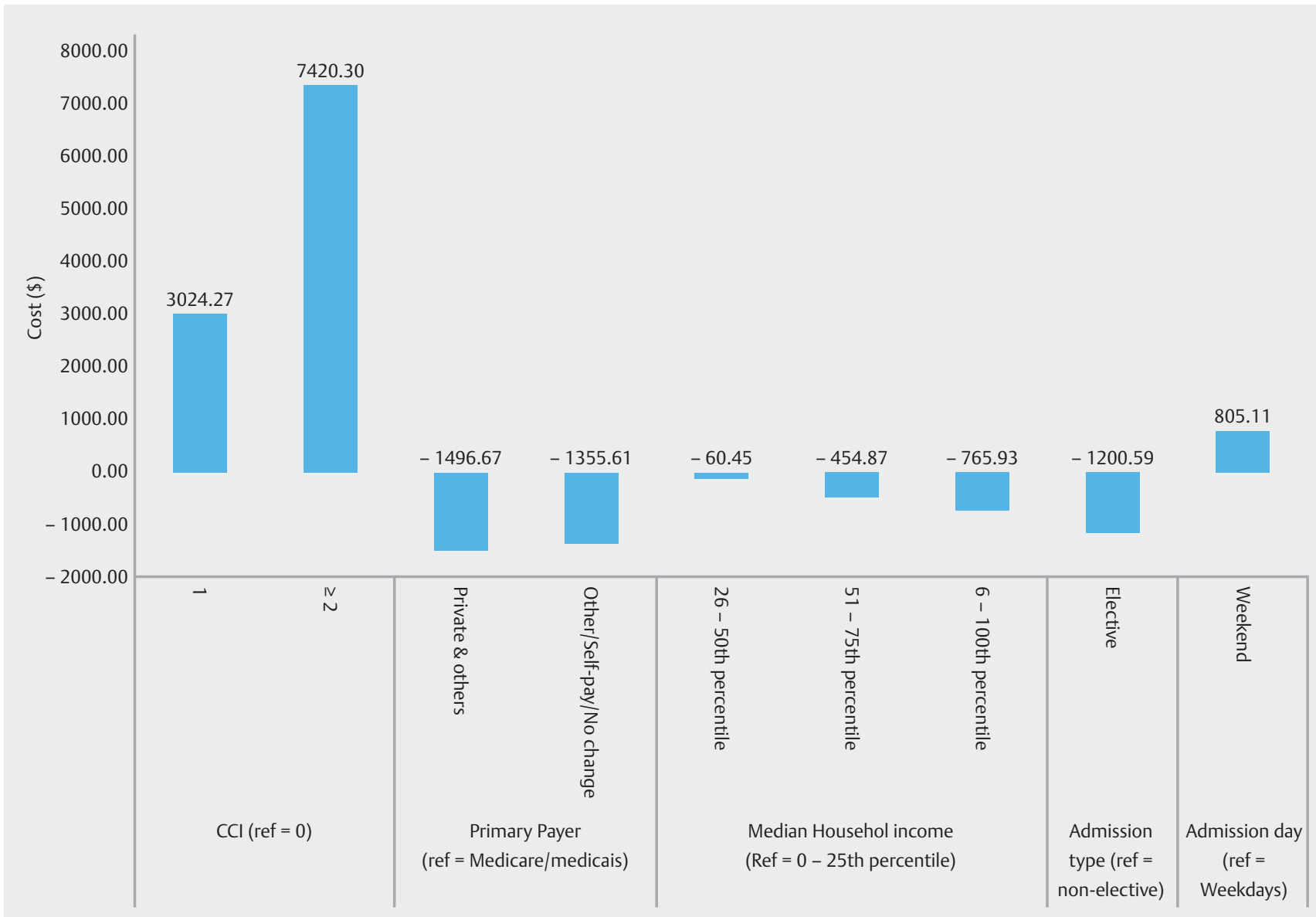

Fig. 3 Multivariate predictors of highest quartile of cost of care for in-hospital ERCP ERCP, endoscopic retrograde cholangiopancreatography 
- Table 4 Multivariate hierarchical linear regression model to identify significant predictors of length of stay.

\begin{tabular}{|c|c|c|c|c|c|}
\hline Variables & $\begin{array}{l}\text { Beta-coefficient } \\
\text { (days) }\end{array}$ & $\begin{array}{l}\text { Std. Error of } \\
\text { Beta (days) }\end{array}$ & $\begin{array}{l}\mathrm{LL} \text { of } 95 \% \mathrm{Cl} \text { of } \\
\text { Beta }(\$)\end{array}$ & $\begin{array}{l}\text { UL of } 95 \% \mathrm{CI} \\
\text { of Beta (\$) }\end{array}$ & $P$ value \\
\hline Age (per 10 years increase) & 0.16 & 17.56 & 0.14 & 0.18 & $<0.001$ \\
\hline \multicolumn{6}{|l|}{ Sex } \\
\hline Male & \multicolumn{5}{|l|}{ Referent } \\
\hline Female & -0.60 & -20.66 & -0.66 & -0.55 & $<0.001$ \\
\hline \multicolumn{6}{|l|}{ Charlson score } \\
\hline 0 & Referent & & & & \\
\hline 1 & 1.15 & 30.62 & 1.08 & 1.23 & $<0.001$ \\
\hline$\geq 2$ & 2.95 & 81.79 & 2.88 & 3.02 & $<0.001$ \\
\hline \multicolumn{6}{|l|}{ Primary Payer } \\
\hline Medicare or Medicaid & \multicolumn{5}{|l|}{ Referent } \\
\hline Private including HMOs \& PPOs & -0.85 & -23.77 & -0.91 & -0.78 & $<0.001$ \\
\hline Other/self-pay/no charge & -0.58 & -10.42 & -0.68 & -0.47 & $<0.001$ \\
\hline \multicolumn{6}{|c|}{ Median household income category for patient's Zip code } \\
\hline 1. 0 -25th percentile & \multicolumn{5}{|l|}{ Referent } \\
\hline 2. $26-50$ th percentile & -0.11 & -2.54 & -0.19 & -0.02 & 0.011 \\
\hline 3. $51-75$ th percentile & -0.28 & -6.34 & -0.37 & -0.19 & $<0.001$ \\
\hline 4. 76 - 100th percentile & -0.35 & -7.11 & -0.44 & -0.25 & $<0.001$ \\
\hline \multicolumn{6}{|l|}{ Admission Type } \\
\hline Non-elective & \multicolumn{5}{|l|}{ Referent } \\
\hline Elective & -0.46 & -22.61 & -0.50 & -0.42 & $<0.001$ \\
\hline \multicolumn{6}{|l|}{ Admission Day } \\
\hline Weekdays & \multicolumn{5}{|l|}{ Referent } \\
\hline Weekends & 0.22 & 6.31 & 0.15 & 0.29 & $<0.001$ \\
\hline \multicolumn{6}{|c|}{ Bed size of Hospital depending on Location \& Teaching Status } \\
\hline Small & \multicolumn{5}{|l|}{ Referent } \\
\hline Medium & 0.01 & 0.08 & -0.17 & 0.18 & 0.935 \\
\hline Large & 0.34 & 3.84 & 0.17 & 0.52 & $<0.001$ \\
\hline \multicolumn{6}{|l|}{ Hospital Region } \\
\hline Northeast & \multicolumn{5}{|l|}{ Referent } \\
\hline Midwest & -1.32 & -12.26 & -1.53 & -1.11 & $<0.001$ \\
\hline South & -0.67 & -6.81 & -0.86 & -0.48 & $<0.001$ \\
\hline West & -1.54 & -10.97 & -1.82 & -1.27 & $<0.001$ \\
\hline \multicolumn{6}{|l|}{ Hospital Location } \\
\hline Rural & \multicolumn{5}{|l|}{ Referent } \\
\hline Urban & 0.87 & 8.47 & 0.67 & 1.07 & $<0.001$ \\
\hline \multicolumn{6}{|l|}{ Hospital Teaching Status } \\
\hline Non-teaching & \multicolumn{5}{|l|}{ Referent } \\
\hline Teaching & 0.40 & 6.19 & 0.28 & 0.53 & $<0.001$ \\
\hline
\end{tabular}


- Table 5 Multivariate hierarchical linear regression model to identify significant predictors of cost of care.

\begin{tabular}{|c|c|c|c|c|c|}
\hline Variables & $\begin{array}{l}\text { Beta-coefficient } \\
\text { (\$) }\end{array}$ & $\begin{array}{l}\text { Std. Error of } \\
\text { Beta (\$) }\end{array}$ & $\begin{array}{l}\text { LL of } 95 \% \mathrm{Cl} \\
\text { of Beta (\$) }\end{array}$ & $\begin{array}{l}\text { UL of } 95 \% \mathrm{CI} \\
\text { of Beta (\$) }\end{array}$ & $P$ value \\
\hline Age (per 10 years increase) & 179.15 & 6.24 & 122.93 & 235.38 & $<0.001$ \\
\hline \multicolumn{6}{|l|}{ Sex } \\
\hline Male & \multicolumn{5}{|l|}{ Referent } \\
\hline Female & -2406.10 & -26.61 & -2583.29 & -2228.91 & $<0.001$ \\
\hline \multicolumn{6}{|l|}{ Charlson score } \\
\hline 0 & \multicolumn{5}{|l|}{ Referent } \\
\hline 1 & 3024.27 & 25.97 & 2796.02 & 3252.52 & $<0.001$ \\
\hline$\geq 2$ & 7420.30 & 66.72 & 7202.34 & 7638.27 & $<0.001$ \\
\hline \multicolumn{6}{|l|}{ Primary payer } \\
\hline Medicare or Medicaid & \multicolumn{5}{|l|}{ Referent } \\
\hline Private including HMOs \& PPOs & -1496.67 & -13.61 & -1712.20 & -1281.15 & $<0.001$ \\
\hline Other/self-pay/no charge & -1355.61 & -7.91 & -1691.63 & -1019.59 & $<0.001$ \\
\hline \multicolumn{6}{|c|}{ Median household income category for patient's zip code } \\
\hline 1. 0 -25th percentile & \multicolumn{5}{|l|}{ Referent } \\
\hline 2. 26 -50th percentile & -64.45 & -0.48 & -325.72 & 196.83 & 0.629 \\
\hline 3. 51 - 75th percentile & -454.87 & -3.29 & -726.04 & -183.69 & 0.001 \\
\hline 4. 76 - 100th percentile & -765.93 & -5.00 & -1065.89 & -465.97 & $<0.001$ \\
\hline \multicolumn{6}{|l|}{ Admission type } \\
\hline Non-elective & \multicolumn{5}{|l|}{ Referent } \\
\hline Elective & -1200.59 & -19.15 & -1323.47 & -1077.70 & $<0.001$ \\
\hline \multicolumn{6}{|l|}{ Admission day } \\
\hline Weekdays & \multicolumn{5}{|l|}{ Referent } \\
\hline Weekends & 805.11 & 7.50 & 594.83 & 1015.38 & $<0.001$ \\
\hline \multicolumn{6}{|c|}{ Bed size of hospital depending on location \& teaching status } \\
\hline Small & \multicolumn{5}{|l|}{ Referent } \\
\hline Medium & -1485.19 & -4.72 & -2101.47 & -868.91 & $<0.001$ \\
\hline Large & -1254.90 & -3.84 & -1895.12 & -614.69 & $<0.001$ \\
\hline \multicolumn{6}{|l|}{ Hospital region } \\
\hline Northeast & \multicolumn{5}{|l|}{ Referent } \\
\hline Midwest & -2729.36 & -5.83 & -3647.64 & -1811.07 & $<0.001$ \\
\hline South & -2958.71 & -6.75 & -3818.29 & -2099.14 & $<0.001$ \\
\hline West & -1467.87 & -2.46 & -2637.47 & -298.28 & 0.014 \\
\hline \multicolumn{6}{|l|}{ Hospital location } \\
\hline Rural & \multicolumn{5}{|l|}{ Referent } \\
\hline Urban & 298.13 & 0.77 & -459.53 & 1055.80 & 0.441 \\
\hline \multicolumn{6}{|l|}{ Hospital teaching status } \\
\hline Non-teaching & \multicolumn{5}{|l|}{ Referent } \\
\hline Teaching & 659.23 & 2.69 & 179.30 & 1139.16 & 0.007 \\
\hline
\end{tabular}


$=105)$ in 2002 to $18,219(\mathrm{SE}=87)$ in $2013(P$ for trend $<0.001)$ ( Table 5).

\section{Discussion}

Our study reflects contemporary data summarizing the overall trend for in-hospital ERCP over a 10-year period from 2002 to 2013. Our main findings are as follows: (1) We noted a shift in paradigm towards increasing trend for therapeutic ERCP; (2) There was a dramatic decline in diagnostic ERCPs; (3) ERCPrelated complications are minimal with low percentage of any serious complications, thus making it a relatively safe procedure; and (4) Inpatient cost of care and LOS decreased over the years during our study period, thus making it an affordable procedure.

A prior study done by Mazen et al, showed a dramatic increase in the trend for overall ERCP between 1988 to 1996, followed by a dramatic drop from 1996 to 1998, which gradually leveled off by 2002 [5]. Since then ours has been the first study examining inpatient ERCPs performed for all indications. Our results are in accordance with most of the population-based studies that show steadily increasing trends in ERCP mostly driven by its therapeutic utilization and a significant drop in its diagnostic utility [6]. MRCP has led to better clinical decisionmaking by gastroenterologists, thus decreasing recommendations for ERCP [7]. Widespread availability of EUS has further led to this decline [8]. Some of the factors related to the decline in diagnostic ERCPs include the risk of post-ERCP pancreatitis, which ranges from $1.6 \%$ to $15.7 \%$ and can be avoided with equally sensitive modalities like EUS and MRCP without the added risk of pancreatitis [9].

Our results demonstrated that most hospitalized patients who underwent ERCPs were older, female and predominantly white, followed by an increasing trend for ERCP among the Hispanic population. This could be explained by the predominance of biliary-pancreatic disease in the previously mentioned population $[10,11]$.

Several studies have proved ERCP to be a safe and effective outpatient procedure when performed with a selective policy [12]. A few studies also showed that most ERCP complications occur within 4 to 6 hours after the procedure, thus observation alone has shown to decrease re-hospitalization rates [13-16]. All of these factors have led to a shift in ERCP from the inpatient to the ambulatory care setting, contributing to the decline in hospital costs. Our results for in-hospital costs are in sync with the above studies.

Numerous published studies in the past have showed a "weekend effect" for various acute conditions like gastrointestinal hemorrhage, where patients admitted on weekends had worse clinical outcomes, higher hospital charges and longer LOS [17 - 19]. ERCP is a technically challenging and resource-intensive procedure that may require coordination of gastroenterologists, trained nursing staff and sometimes anesthesia services, which is difficult to obtain during the weekends. Our study similarly shows an increased cost and LOS for admissions during the weekends as compared to the weekdays.
Most of the limitations of our study were inherent in use of an administrative database (NIS), which lacks the details available in trials and registries. The accuracy of coding may be questionable. Long-term outcomes, complications and re-hospitalization rates could not be assessed. We could not consider individual provider volume and could not assess rate of failure or re-hospitalization due to the use of the same diagnostic codes for both indications and complications of the procedure, like pancreatitis and cholangitis. The outcome of the procedure, degree of procedural difficulties, and endoscopy experience could not be assessed. We also could not assess the procedures conducted in the outpatient settings, which contributes to a significant number in the current era.

\section{Conclusions}

In summary, our study represents one of the first large-scale population based study in the United States conducted in the past decade. Our study indicates a significant decline in the diagnostic indications for inpatient ERCP and a steady rise in therapeutic ERCPs. This could be explained by the increasing prevalence of gall stone disease, ever evolving indications for ERCP and also the rise in safer diagnostic alternatives like endoscopic ultrasound and magnetic resonance cholangiopancreatography. Most common therapeutic procedures in our study included endoscopic sphincterotomy, followed by endoscopic biliary stone removal and endoscopic biliary stenting. Pancreatitis, biliary obstruction and cholangitis were the top 3 commonly coded diagnoses for ERCP, however, our data set prevents us from determining whether pancreatitis and cholangitis were the cause for ERCP or if these were post-procedural complications. We also identified a trend in decreasing cost for ERCP hospitalizations during our study period. That may be due to increasing elective, outpatient management of non-urgent ERCPs. Avoiding delays in weekend procedures can help us to further reduce the cost and the LOS in the hospital.

\section{Competing interests}

None

References

[1] [Anonymous] NIH state-of-the-science statement on endoscopic retrograde cholangiopancreatography (ERCP) for diagnosis and therapy. NIH Consens State Sci Statements 2002; 19: 1- 26

[2] Adler DG, Baron TH, Davila RE et al. ASGE guideline: the role of ERCP in diseases of the biliary tract and the pancreas. Gastrointest Endosc 2005; 62: $1-8$

[3] Sood N, Huckfeldt P], Escarce J] et al. Medicare's bundled payment pilot for acute and postacute care: analysis and recommendations on where to begin. Health Aff (Millwood) 2011; 30: 1708-1717

[4] Healthcare Cost and Utilization P. HCUP Facts and Figures. In, HCUP Facts and Figures: Statistics on Hospital-Based Care in the United States, 2007. Rockville (MD): Agency for Healthcare Research and Quality (US); 2009 
[5] Mazen Jamal M, Yoon E], Saadi A et al. Trends in the utilization of endoscopic retrograde cholangiopancreatography (ERCP) in the United States. Am J Gastroenterol 2007; 102: 966 - 975

[6] Coelho-Prabhu N, Shah ND, Van Houten $\mathrm{H}$ et al. Endoscopic retrograde cholangiopancreatography: utilisation and outcomes in a 10year population-based cohort. BMJ Open 2013; 3: DOI 10.1136/ bmjopen-2013-002689

[7] Akisik MF, Jennings SG, Aisen AM et al. MRCP in patient care: a prospective survey of gastroenterologists. AJR Am J Roentgenol 2013; 201: $573-577$

[8] Sahel J, Barthet M, Gasmi M. The substitution of endoscopic ultrasonography for endoscopic retrograde cholangio-pancreatography: implications for service development and training. Eur J Gastroenterol Hepatol 2004; 16: 291 - 294

[9] Cotton PB, Garrow DA, Gallagher ] et al. Risk factors for complications after ERCP: a multivariate analysis of 11,497 procedures over 12 years. In, Gastrointest Endosc. United States2009: $80-88$

[10] Everhart JE, Khare M, Hill M et al. Prevalence and ethnic differences in gallbladder disease in the United States. In, Gastroenterology. United States 1999: 632-639

[11] Maurer KR, Everhart JE, Ezzati TM et al. Prevalence of gallstone disease in Hispanic populations in the United States. Gastroenterology 1989; 96: $487-492$

[12] Jeurnink SM, Poley JW, Steyerberg EW et al. ERCP as an outpatient treatment: a review. Gastrointest Endosc 2008; 68: 118-123
[13] Freeman ML, Nelson DB, Sherman S et al. Same-day discharge after endoscopic biliary sphincterotomy: observations from a prospective multicenter complication study. The Multicenter Endoscopic Sphincterotomy (MESH) Study Group. Gastrointest Endosc 1999; 49: 580 586

[14] Hui CK, Lai KC, Wong WM et al. Outpatients undergoing therapeutic endoscopic retrograde cholangiopancreatography: six-hour versus overnight observation. J Gastroenterol Hepatol 2004; 19: 1163-1168

[15] Katsinelos P, Kountouras ], Chatzimavroudis G et al. Outpatient therapeutic endoscopic retrograde cholangiopancreatography is safe in patients aged 80 years and older. Endoscopy 2011; 43: 128-133

[16] Duncan HD, Hodgkinson L, Deakin M et al. The safety of diagnostic and therapeutic ERCP as a daycase procedure with a selective admission policy. Eur J Gastroenterol Hepatol 1997; 9: 905-908

[17] Shaheen AA, Kaplan GG, Myers RP. Weekend versus weekday admission and mortality from gastrointestinal hemorrhage caused by peptic ulcer disease. Clin Gastroenterol Hepatol 2009; 7: 303-310

[18] Worni M, Schudel IM, Ostbye T et al. Worse outcomes in patients undergoing urgent surgery for left-sided diverticulitis admitted on weekends vs weekdays: a population-based study of 31832 patients. Arch Surg 2012; 147: 649-655

[19] Palmer WL, Bottle A, Davie C et al. Dying for the weekend: a retrospective cohort study on the association between day of hospital presentation and the quality and safety of stroke care. Arch Neurol 2012; 69: $1296-1302$ 